\title{
Determination of Visual Binary Orbits from Short-Arc Observations Using the Method of Apparent Motion Parameters ${ }^{1}$
}

\author{
A. A. KISSELEV \\ The Main Astronomical Observatory of Russian Academy of Sciences, \\ Pulkovo, St. Petersburg 196140, Russia
}

\begin{abstract}
The key formulae of the AMP-method for deriving the orbital elements of wide visual double stars are considered. The usual astrometrical observational data must be supplemented with the values of radius of curvature of observed arc of the binary, its trigonometric parallax and the astrophysical data: the sum of the mass of the components and their relative radial velocity. Thus, the orbital elements can be derived on the basis of short-arc observations.
\end{abstract}

There are hundreds of wide visual double stars observed since W. Struve's time. The periods of revolutions of these stars exceed 500 years. The orbital elements of these binaries cannot be determined by classical methods, based on the positional observations exclusively. But there is a good chance to solve the problem if the astrometric data would be supplemented by selected data from astrophysics. In this paper, we consider a set of formulae which ought to help us in the problem of computation of orbital elements of a double star on the basis of short-arc observations, that is to say, we deal with the wide binaries with long and very-long periods of revolution.

We assume that for a given double star $A B$ the following observational parameters are deduced with sufficient accuracy.

- The astrometric parameters:

$\rho, \theta=$ angular distance and position angle of $\mathrm{AB}$

$\mu, \psi=$ angular motion of $\mathrm{B}$ relative to $\mathrm{A}$ and its position angle - both defined by (1)

$$
\mu=\sqrt{\dot{\rho}^{2}+(\rho \dot{\theta})^{2}} ; \psi=\theta+(\operatorname{sign} \dot{\theta}) 90^{\circ}-\arctan \frac{\dot{\rho}}{\rho \dot{\theta}}
$$

$\rho_{c}=$ radius of the curvature of the short arc of the apparent orbit.

The above parameters correspond approximately to the given moment in the middle of the observational time interval.

$\pi_{t}=$ the trigonometrical parallax.

- The astrophysical parameters:

$V_{r}=V_{r B}-V_{r A}=$ the radial velocity of $\mathrm{B}$ relative to $\mathrm{A}$.

$M_{A B}=$ the sum of mass components $\mathrm{A}$ and $\mathrm{B}$ in units of solar mass.

\footnotetext{
${ }^{1}$ The author of this paper was unable to attend the Colloquium - eds.
} 


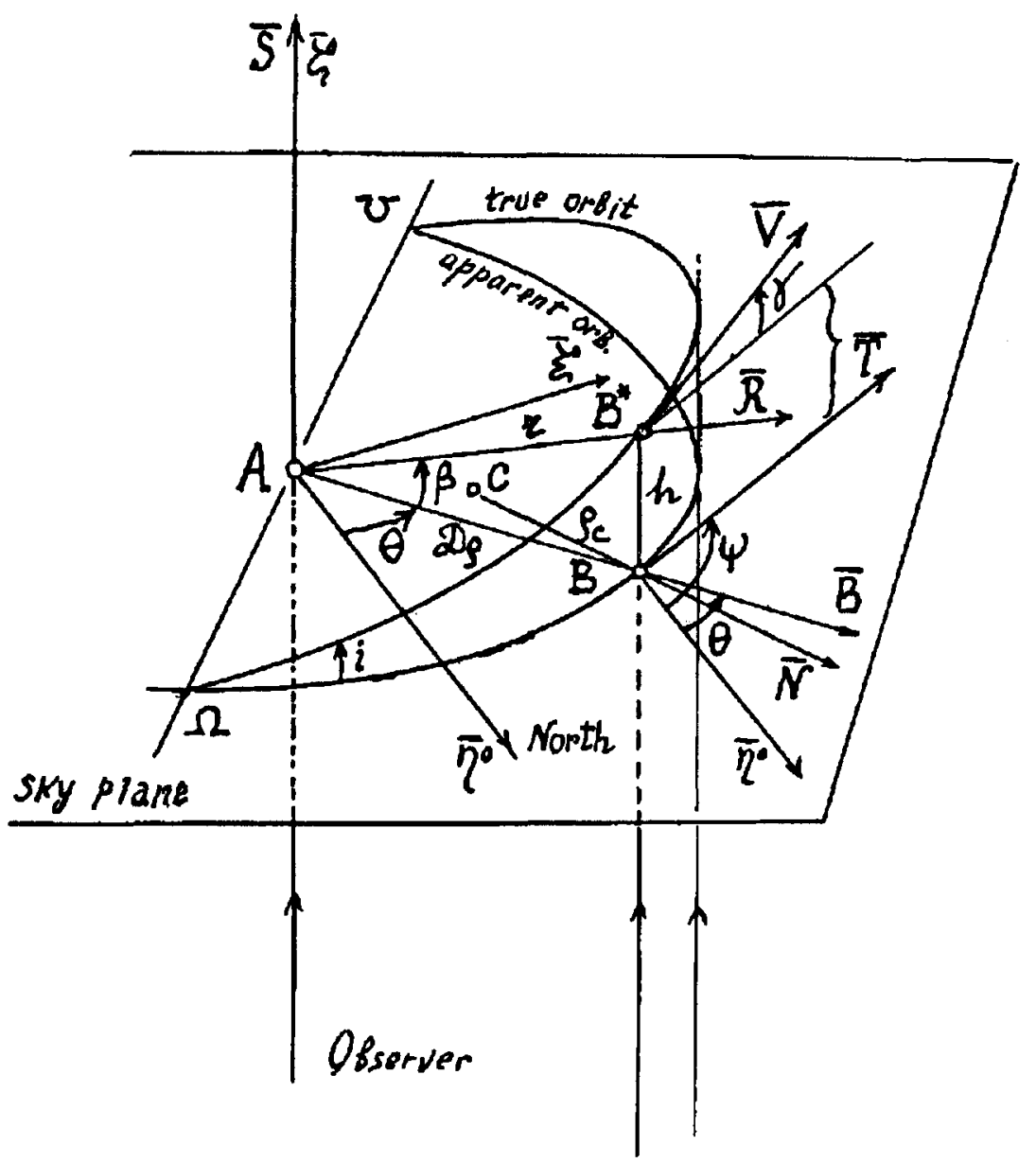

FIGURE 1. Vector designations used in the AMP formulae

The apparent and true motions of the components of a double star are connected by three vector-differential equations $(2,3,4)$, that point on the dynamic nature of the problem.

$$
\begin{gathered}
\bar{r}=r \bar{R}=D \rho \bar{B}+h \bar{S} \\
\dot{\bar{r}}=v \bar{V}=D \mu \bar{T}+\dot{h} \bar{S} \\
\ddot{\bar{r}}=-\kappa^{2} \frac{\bar{R}}{r^{2}}=(D \dot{\mu}+2 \dot{h} \mu) \bar{T}-(\operatorname{sign} \dot{\theta}) D \frac{\mu^{2}}{\rho_{c}} \bar{N}+\ddot{h} \bar{S}
\end{gathered}
$$

Here we use the following designations (see Figure 1):

$$
\bar{S}=\text { the unit vector of the directions } \overline{O A}\|\overline{A S}\| \overline{B B^{\star}} \| \bar{\zeta}^{\circ}
$$


$\bar{B}, \bar{T}, \bar{N}=$ the unit vectors on the sky plane, which determines the directions of apparent position and motion $\mathrm{B}$ relative to $\mathrm{A}$; where $\bar{N}$ is the direction of normal $(\bar{N} \perp \bar{T})$.

$\bar{r}, \dot{\bar{r}}, \ddot{\bar{r}}=$ the spatial vectors $\overline{A B^{\star}}$ and their derivatives.

$\bar{R}, \bar{V}=$ the unit vectors of $\bar{r}$ and $\dot{\bar{r}}$,

$\mathrm{D}=$ the range of the binary $\mathrm{AB}$ to the observer.

$$
D=\left(\pi_{t} \sin 1^{\prime \prime}\right)^{-1} A U .
$$

$h, \dot{h}, \ddot{h}=$ the range $\mathrm{B}$ to $\mathrm{B}^{\star}$ and their derivatives

$$
\bar{h}=0.211 V_{r} ;[\dot{h}]=\frac{a u}{y e a r} ;\left[V_{r}\right]=\frac{k m}{s}
$$

$\kappa^{2}=$ the dynamic constant of the astrocentric motion; its value is

$$
\kappa^{2}=4 \pi^{2} M_{A B} \frac{(a u)^{3}}{(\text { year })^{2}}
$$

If the equation system $(2-4)$ is solved, we can find vectors $r \bar{R}$ and $v \bar{V}$ with respect to the astrocentric coordinate axes $\left(A \bar{\xi}^{\star} \bar{\eta}^{\circ} \bar{r}^{\circ} \bar{\Sigma}^{\circ}\right)$. Then the orbital elements of the binary can be calculated in the usual celestial mechanics manner. We suggest the following solution for equation (2-4), using a complete set of observational parameters.

(1.) From the third equation we have:

$$
r^{3}=\kappa^{2} \frac{\rho \rho_{c}}{\mu^{2}} \bar{B} \bar{N}= \pm \kappa^{2} \frac{\rho \rho_{c}}{\mu^{2}} \sin (\Psi-\theta)
$$

This fundamental relation allows us to compute in the range $A$ to $B^{\star}$ in astronomical units, using the assumed value of $M_{A B}$ and the astrometric data, where the value of curvature radius is of essential importance.

(2.) We obtain $\beta$, the angle between $\bar{R}$ and the sky plane, and the value of $\mathrm{h}$ :

$$
\cos \beta=D \rho / r ; h= \pm \sqrt{r^{2}-(D \rho)^{2}}
$$

These relations offer us a good control over the values involved, since

$$
D \rho=\rho / \pi_{t} \leq r
$$

(3.) We also obtain $\gamma$, the angle between $\bar{V}$ and the sky plane:

$$
\tan \gamma=\frac{\dot{h}}{D \mu}=\frac{V_{\mathrm{r}}}{4.74 D \mu}
$$


(4.) The results (9) and (11) enable us to construct the unit vectors $\mathbf{R}$ and V, as referred to the axes of astrocentric coordinates $A \bar{\xi}^{\circ} \bar{\eta}^{\circ} \bar{r}^{\circ}$

$$
r \bar{R}=\left\{\begin{array}{c}
\sin \theta \cos \beta \\
\cos \theta \cos \beta \\
\pm \sin \beta
\end{array} \quad ; \quad \bar{V}=\left\{\begin{array}{c}
\sin \Psi \cos \gamma \\
\cos \Psi \cos \gamma \\
\sin \gamma
\end{array}\right.\right.
$$

Vectors $\bar{R}$ and $\bar{V}$ will be essential to derive the geometrical elements of the orbit to be found.

(5.) The value of the spatial velocity $v$ is given by the known parameters

$$
v=\sqrt{(D \mu)^{2}+\dot{h}^{2}}
$$

(6.) Finally, we compute the dynamical elements of the orbit in question:

$$
\begin{aligned}
& a=\left(2 / r-v^{2} / \kappa^{2}\right)^{-1} \\
& P=\sqrt{a^{3} / M_{A B}}
\end{aligned}
$$

Formula (15) affords us a strict control over the dynamical state of the binary star under consideration.

It is obvious that in the general case using the AMP-method we come to two orbits which are identical in dynamical meaning but differ in geometrical one.

The set of the above formulae we regard as the algorithm of the AMPmethod (Kisselev \& Kijaeva 1980), assigned to examine kinematics and dynamics of the selected wide visual couple in the vicinity of the Sun.

The AMP-method serves as a basic tool in the investigations of double stars at Pulkovo observatory which have been carried out with 26 -inch Zeiss astrograph since 1960. In the course of these investigations (Kisselev et al. 1990) we have determined 10 new orbits of wide binaires, three of them were computed for the first time (Kisselev et al. 1990, Kisselev \& Kijaeva 1988). Some of these orbits were used to examine the problem of dark-companion existence (see 0.V. Kijaeva, this Colloquium).

\section{REFERENCES}

Kisselev A.A. \& Kijaeva O.V., 1980, Astr. J. USSR, 57, 6, 1227

Kisselev A.A. \& Kijaeva O.V., 1988, Ap\&SS, 142, 181

Kisselev A.A. et al. 1990, in Inertial Coordinate System on the Sky, IAU Symp. 141, 63 